\title{
A NEW METHOD FOR THE SIMULATION OF ALLOYS: APPLICATION TO INTERFACIAL SEGREGATION
}

\author{
R. NAJAFABADI ${ }^{1}$, H. Y. WANG', D. J. SROLOVITZ ${ }^{1}$ and R. LeSAR ${ }^{2}$ \\ 'Department of Materials Science and Engineering, University of Michigan, Ann Arbor, MI 48109 \\ and ${ }^{2}$ Theoretical Division, Los Alamos National Laboratory, Los Alamos, NM 87545, U.S.A.
}

\author{
(Received 14 March 1991; in revised form 16 May 1991)
}

\begin{abstract}
We present a new, accurate method for determining the properties of defects in alloys at finite temperature, including equilibrium segregation. This method is based upon a point approximation for the configurational entropy, an Einstein model for vibrational contributions to the free energy and may be employed with any type of description of atomic interactions. The atomic structure, segregation and thermodynamics of a defect in an alloy is determined by minimizing the free energy with respect to atomic coordinates and composition of each site at constant chemical potential. In order to test the accuracy of this approach, we compare our results with accurate Monte Carlo determinations. Overall, very good agreement for segregation to free surfaces and grain boundaries in $\mathrm{Cu}-\mathrm{Ni}$ alloys is obtained. One of the main advantages this new method enjoys over other methods such as Monte Carlo, is the efficiency with which the atomic structure of a defect, segregation and thermodynamic properties can be determined. This efficiency is obtained in the framework of a very straightforward method and with little loss in accuracy.
\end{abstract}

Reaume - Nous présentons une méthode nouvelle et précise pour déterminer les propriétés des défauts dans les alliages à une température donnée, y compris la ségrégation à l'équilibre. Cette méthode est basée sur une approximation par points de l'entropie de configuration, un modèle d'Einstein pour les contributions vibrationnelles à l'énergie libre, et elle peut être utilisée pour n'importe quel type de description des interactions atomiques. La structure atomique, la ségrégation et la thermodynamique d'un défaut dans un alliage sont déterminées en minimisant l'énergie libre par rapport aux coordonnées atomiques et à la composition de chaque site à potentiel chimique constant. Pour tester la précision de cette approche, nous avons comparé nos résultats à des déterminations de Monte Carlo précises. L'accord est très bon pour la ségrégation vers les surfaces libres et les joints de grains dans les alliages $\mathrm{Cu}-\mathrm{Ni}$. L'un des principaux avantages offerts par cette nouvelle méthode, par rapport à d'autres méthodes telles que celle de Monte Carlo, est l'efficacité avec laquelle on peut déterminer la structure atomique d'un défaut, la ségrégation et les propriétés thermodynamiques. Cette efficacité est obtenue dans le cadre d'une méthode très directe et en perdant peu de précision.

\begin{abstract}
Zasammenfaseung-Wir stellen eine neue genaue Methode zur Bestimmung der Defekteigenschaften in Legierungen bei endlichen Temperaturen einschließlich der Gleichgewichtssegregation vor. Dieses Verfahren beruht auf einer Punktnäherung für die Konfigurationsentropie, einem Einstein-Modell der Schwingungsbeiträge zur freien Energie, und kann bei jeder Beschreibungsart der atomaren Wechselwirkung benutzt werden. Atomare Struktur, Segregation und Thermodynamik eines Defektes werden durch Minimalisierung der freien Energie hinsichtlich der Atomkoordinaten und der Zusammensetzung jedes Ortes bei konstantem chemischen Potential bestimmt. Um die Genauigkeit dieser Näherung zu bestimmen, vergleichen wir unsere Ergebnisse mit genauen Monte-Carlo-Simulationen. Insgesamt stimmen die Ergebnisse für Segregation an freie Oberflächen und an Korngrenzen in $\mathrm{Cu}-\mathrm{Ni}$-Legierungen sehr gut überein. Einer der Hauptvorteile dieser neuen Methode gegenüber anderen, wie etwa der Monte-CarloRochnungen, ist die Effektivität, mit der die atomare Struktur eines Defektes, die Segregation und die thermodynamischen Eigenschaften bestimmt werden kann. Diese wird im Rahmen eines sehr einfachen Verfahrens ohne großen Genauigkeitsverlust erreicht.
\end{abstract}

\section{INTRODUCTION}

Interfaces in solids are known to play crucial roles in a wide variety of physical phenomena. Grain boundaries in polycrystalline materials, for example, influence such properties as strength, toughness, electrical resistivity, band structure, diffusivity, etc. The properties of free surfaces control phenomena such as catalysis. Small changes in the composition of a material are known to produce large changes in those physical phenomena which are controlled by interfaces. This magnification effect can be attributed, in many cases, to interfacial segregation, which results in very different bulk and interface concentrations. Segregation to grain boundaries can $\mathrm{em}$ brittle a material, change its diffusivity, modify its resistance to stress corrosion resistance, etc. Segregation to free surfaces can produce devastating or highly beneficial effects in catalysis. Since intergranular fracture in brittle materials replaces a grain 
boundary with free surfaces, both the tendency of an impurity to segregate to grain boundaries and free surfaces dictate impurity effects in fracture. Impurities that segregate to grain boundaries but not to free surfaces tend to enhance fracture resistance. An understanding of such interfacial phenomena thus requires a knowledge of not only the structure of the interface, but the degree of compositional change due to segregation and, in turn, the effect of that change on the thermodynamics of the interface. The focus of the present work is the development of simple, yet accurate, theoretical techniques to calculate the structure, composition and thermodynamics of interfaces in alloyed materials.

A number of different simulation techniques have been developed to determine the structure of interfaces in pure materials and in situations where the composition does not change. For these cases at $0 \mathrm{~K}$, static relaxation techniques can be used by minimizing the internal energy with respect to atomic coordinates to obtain the stable interfacial structure and energy at $T=0 \mathrm{~K}$. At $T>0$, molecular dynamics (MD) and Monte Carlo (MC) methods may be employed to determine interfacial structure in these compositionally fixed structures. Recently, we introduced an alternative approach [1], based on a simple description of the free energy of the material, for finite-temperature simulations of interfacial structure and properties that requires a simple energy relaxation, instead of a full-scale simulation. This method, called the local-harmonic ( $\mathrm{LH}$ ) model, is based on a simple harmonic description of the atomic vibrations, and has proven quite successful $[2,3]$ in describing the finite-temperature structure and thermodynamic properties of grain boundaries. Additionally, this method is computationally efficient compared with other finite temperature simulation methods such as MC or MD.

The study of segregation to interfaces is less well developed. Simple, lattice-gas models have been used $[4,5]$, but these suffer from an unrealistic treatment of the interactions between the particles and the elastic properties of the material, though they do allow for fairly straightforward theoretical analysis. Recent advances in Monte Carlo simulation methods $[6,7]$ have allowed this method to be extended to alloy systems where the local composition can change during the course of the simulation. This has led to the first atomistic studies of equilibrium segregation to interfaces. While this method does yield equilibrium interfacial structure and composition, it has never been successfully applied to obtaining thermodynamic information. Unfortunately, these calculations require very substantial computational resources and hence this method is generally limited to supercomputer applications. Our goal in studying interfacial phenomena is not simply to elucidate the properties of individual interfaces under well defined conditions, but is, instead, directed at understanding the trends in interfacial and segregation behavior as a function of the experimental parameters; temperature, bulk composition, and interface geometry/ crystallography. In order to elucidate trends, computational efficiency is not simply a matter of convenience but determines whether such studies are possible. Given this goal, we conclude that Monte Carlo methods do not yield sufficient information and are computationally too demanding for systematic studies of interfacial properties in alloys.

The goal of the present work is to develop simple techniques that obviate massive computer simulations yet that are accurate enough to be useful. By accurate, we mean that the results should produce the proper trends in interfacial behavior and are at least semiquantitative, which is the most that can be asked of any atomistic simulation procedure given the empirical/approximate nature of the interatomic potentials. We approach this problem based on the use of the LH model, which is known to be quite accurate for pure materials. Extension of this method to alloy systems is achieved by introducing effective (or mean-field) atoms, that have properties that are a concentration-weighted mix of the different atom types. The LH model then can be used with essentially the same ease and efficiency as for pure systems. In addition, the extension of the method to alloys requires the addition of some description of the configurational entropy. There are a number of methods, such as the cluster variation method [8], the Bethe method [9], etc. which have had some successes in determining the configurational entropy of perfect crystals. Unfortunately, these methods are rather difficult to apply to nonuniform systems and with non-pairwise interactions. We thus choose to use a simpler approximation, namely that derived assuming ideal mixing. The configurational entropy in this approximation reduces to a simple point term that depends only on the concentration of each component at each site and that neglects any site-site correlations. Though this is a very simple approximation, we demonstrate below that it works rather well for studying defects in alloys at temperatures not too close to transformation temperatures.

In the present paper, we first describe our new simulation approach and then apply it to segregation at grain boundaries and free surfaces in the $\mathrm{Cu}-\mathrm{Ni}$ system. These systems were chosen because accurate results for these cases are available from Monte Carlo calculations employing the same description of the interatomic interaction as used here. Any discrepancies between our results and those from the Monte Carlo calculations are thus due to the approximations in our methods. In the present study and the Monte Carlo calculations, the atomic interactions are described within the framework of the embedded-atom model (EAM) [10], in which the energy of a metal is expressed as a sum of pairwise repulsive terms and a many-body term that approximates the energy to embed an atom in the electron gas. 


\section{METHOD}

The goal of the present study is to develop an accurate, efficient method to study the structure, composition and thermodynamics of defects in alloy systems. The approach we take in reaching this goal is to define an approximate expression for the free energy of a multicomponent atomic system and minimize that free energy with respect to the atomic coordinates and the compositional profile in the material. The free energy of a multicomponent system consists of several distinguishable parts. These include atomic bonding, atomic vibrations and configurational entropy (i.e. the entropy associated with the relative spatial distribution of the atomic species). In the present study, we describe atomic bonding using standard atomistic simulation methods (for metals, we employ the Embedded Atom Method (EAM) potentials [6, 11]). The effects of atom vibrations are included within the framework of the Local Harmonic (LH) model [1], which we previously introduced. Configurational entropy is described on the basis of a point approximation. While each of these features can, in principle, be described more accurately than we are doing here, each has drawbacks that prevent their application to efficient atomistic simulations. For example, electronic structure calculations, diagonalization of full dynamical matrices and the cluster variation method provide very accurate descriptions of atomic bonding, atomic vibrations and configurational entropy, respectively, but generally do so at such great computational expense that they are impractical for atomistic simulations. In the remainder of this section, we review the Local Harmonic description of atomic vibrations, introduce the point approximation for configurational entropy in a form appropriate for atomistic simulations, and then finally describe the method we employ in examining interfacial segregation.

The local harmonic (LH) model has been applied with considerable success to perfect and defected solids of single elements $[2,3]$. To a good approximation, the atoms in a solid can be treated as harmonic oscillators. We simplify the harmonic approximation further by neglecting all terms that couple vibrations of different atoms. For a perfect crystal with one type of atom, this becomes the Einstein model. Here we are mainly concerned with defects, so that each atom potentially has different vibrational properties. The classical vibrational contribution to the free energy is then given by

$$
A_{\mathrm{v}}=k_{\mathrm{b}} T \sum_{i=1}^{N} \sum_{\beta=1}^{3} \ln \left[\frac{h \omega_{i \beta}}{2 \pi k_{\mathrm{B}} T}\right]
$$

where $k_{\mathrm{B}}$ is the Boltzmann constant, and $\omega_{i 1}, \omega_{i 2}$, and $\omega_{i 3}$ are the three vibrational eigenfrequencies of atom $i$. Neglecting the coupling of vibrations on different atoms, these frequencies may be determined in terms of the local dynamical matrix of each atom
$D_{i k \beta}=\left(\partial^{2} E / \partial x_{i z} \partial x_{i \beta}\right)$, where $E$ is the potential energy determined from summing the interatomic potential and the $x_{i \beta}$ correspond to atomic displacements of atom $i$ in some coordinate system. Diagonalization of this $3 \times 3$ matrix yields the three force constants $k_{i \beta}$ for atom $i$. The vibrational frequencies are then determined as $\omega_{i \beta}=\left(k_{i \beta} / m\right)^{1 / 2}$, where $m$ is the atomic mass. As described in references [1,2], the approximations of the LH model reduces the calculation of the vibrational contribution to the free energy from one of the diagonalizing a $3 N \times 3 N$ dynamical matrix to the calculation of $N$ determinants of $3 \times 3$ matrices, where $N$ is the number of atoms in the system. In our previous applications of the $\mathrm{LH}$ model $[1,2]$, we have demonstrated that the approximations inherent in the LH model lead to errors in the free energy of perfect close-packed metal crystals, which are typically of the order of $1 \%$ at the melting temperature and much less at lower temperatures $[1,3,12]$.

In order to study alloys, it is useful to introduce the concept of an "effective atom". Consider first the case of a binary alloy consisting of $a$ and $b$ atoms. If we were to monitor a particular atom site in such an alloy for a long time, we would find that that atomic site would be occupied by a atoms with probability $c_{\mathrm{a}}$ and by $\mathrm{b}$ atoms with probability $c_{\mathrm{b}}=1-c_{\mathrm{a}}$ (neglecting vacancies). Ignoring the temporal correlation between the site occupancies, we can then define the spatial compositional profile within the alloy crystal as $c_{\mathrm{a}}(r)$. Within this framework, the vibrational free energy of the crystal may be described as in equation (1), with the atomic interactions used to describe the local force constants appropriately averaged (as described below) and the mass of the effective atom employed in the frequency calculations given by $m_{i}=c_{\mathrm{a}}(i) m_{\mathrm{a}}+c_{\mathrm{b}}(i) m_{\mathrm{b}}$. An alternative approach is to determine the vibrational free energy as $A_{\mathrm{v}}=c_{\mathrm{a}}(i) A_{\mathrm{v}}(\mathrm{a})+c_{\mathrm{b}}(i) A_{\mathrm{v}}(\mathrm{b})$, where $A_{\mathrm{v}}(\mathrm{a})$ is given by equation (1) evaluated with the frequencies $\omega_{i \beta}(\mathrm{a})$. Our tests indicate that the two approaches yield nearly the same results. We have adopted the first approach as it is the simpler of the two and is more consistent with the concept of an "effective atom".

In the spirit of the "effective atom" approximation, we assume that the occupation of different atomic sites is uncorrelated. This results in a simple point approximation for the configurational entropy [13]

$$
S_{\mathrm{c}}=-k_{\mathrm{B}} \sum_{i=1}^{N}\left\{c_{a}(i) \ln \left[c_{\mathrm{a}}(i)\right]+c_{\mathrm{b}}(i) \ln \left[c_{\mathrm{b}}(i)\right]\right\}
$$

We note that since this expression always assumes no correlation between site occupancies, it is a rigorous upper bound to the true configurational entropy. In almost all situations, we note that at least some short range correlation does exist. The assumption of no spatial correlations employed in obtaining the configurational entropy [equation (2)] does not imply that spatial correlations will not form in our 
simulations since spatial correlations are predominantly a result of the properties of the atomic interactions.

In the simulations described below, we employ a reduced, or baby, Grand Canonical ensemble, where the total number of atoms remains fixed but the relative amounts of each atomic species varies. The appropriate thermodynamic potential for this type of ensemble is the grand potential and is given by [14]

$$
\begin{aligned}
\Omega=A+\Delta \mu \sum_{i=1}^{N} & c_{\mathrm{a}}(i) \\
& =E+A_{\mathrm{v}}-T S_{\mathrm{c}}+\Delta \mu \sum_{i=1}^{N} c_{\mathrm{a}}(i)
\end{aligned}
$$

where $A$ is the Helmholtz free energy and $\Delta \mu$ is the difference in chemical potential between atoms of type a and $b$. Given $\Delta \mu$, the equilibrium concentration at each site can be determined by minimizing $\Omega$ with respect to those concentrations. This approach was previously employed by Lundberg [15] to study surface segregation. However, he forced all of the atoms in each atomic layer to have the same concentration, thereby leading to a quasi-one dimensional calculation. More importantly, his calculations ignored the often dominant effect of the vibrational contributions to the free energy.

While the above discussion is independent of the method in which atomic bonding is described, it is useful to clarify the application of the "effective atom" approach by way of application to a particular atomic bonding scheme. Since the description of atomic bonding employed in the simulations reported herein were performed within the framework of the Embedded Atom Method (EAM), we have chosen the EAM method for the present discussion. The Embedded-atom model (EAM) potentials have been quite successful in the calculation of bulk and surface properties of both pure metals and binary alloys [see e.g. Ref. 11]. The EAM interaction energy is written as

$$
E=\sum_{i=1}^{N} F_{i}\left(\bar{\rho}_{i}\right)+\frac{1}{2} \sum_{i=1}^{N} \sum_{j \neq i-1}^{N} \Phi_{i j}\left(R_{i j}\right)
$$

where the sum on $i$ is over all $N$ atoms in the system and the sum on $j$ is over all atoms interacting with atom $i, \Phi_{i j}\left(R_{i j}\right)$ is a pair potential between atoms of $i$ and $j$ separated by the distance $R_{i j}$, and $F_{i}\left(\rho_{i}\right)$ is the embedding term that accounts for the energy required to embed atom $i$ in a uniform electron gas with density

$$
\bar{\rho}_{t}=\sum_{j \neq 1} \rho_{j}\left(R_{i}\right)
$$

where $\rho_{j}\left(R_{y}\right)$ is the electron density of atom $j$ evaluated at atom $i$. Note, $F_{i}$ is a nonlinear function that is obtained, in practice, by requiring that the energy of the perfect crystal lattice matches the universal binding curve of Rose et al. [16].
We divide the average interaction energy into two parts, the pair terms and the many-body embedding energy. Neglecting all correlations between the concentration on one site and that on any other site, the contribution to the average energy from the pair terms is given by

$$
\begin{aligned}
E_{\text {pair }}= & \frac{1}{2} \sum_{i=1}^{N} \sum_{j \neq i=1}^{N} \\
& \times\left\{c_{\mathrm{a}}(i) c_{\mathrm{a}}(j) \phi_{\mathrm{aa}}\left(R_{i j}\right)+c_{\mathrm{b}}(i) c_{\mathrm{b}}(j) \phi_{\mathrm{bb}}\left(R_{\mathrm{lj}}\right)\right. \\
& \left.+\left[c_{\mathrm{a}}(i) c_{\mathrm{b}}(j)+c_{\mathrm{b}}(i) c_{\mathrm{a}}(j)\right] \phi_{\mathrm{ab}}\left(R_{\mathrm{l}}\right)\right\} .
\end{aligned}
$$

We approximate the average embedding energy by expanding $\left\langle F_{i}\right\rangle$, where \langle\rangle indicates an ensemble average, about the average density as

$$
\left\langle F_{i}\left(\bar{\rho}_{i}\right)\right\rangle \approx F_{i}\left(\left\langle\bar{\rho}_{i}\right\rangle\right)+\frac{1}{2}\left(\frac{\partial^{2} F_{i}}{\partial \bar{\rho}_{i}^{2}}\right)_{\left\langle\hat{p}_{i}\right\rangle}\left(\left\langle\bar{\rho}_{i}^{2}\right\rangle-\left\langle\bar{\rho}_{i}\right\rangle^{2}\right)
$$

where

$$
\left\langle\bar{\rho}_{i}\right\rangle=\sum_{j \neq l=1}^{N}\left\{c_{\mathrm{a}}(j) \rho_{\mathrm{a}}\left(R_{i j}\right)+c_{\mathrm{b}}(j) \rho_{\mathrm{b}}\left(R_{i j}\right)\right\} .
$$

We find that the ratio of the second term in equation (7) to the first is typically about $10^{-5}$ for the system studied here. Therefore, we approximate $\left\langle F_{l}\left(\bar{\rho}_{i}\right)\right\rangle$ with $F_{i}\left(\left\langle\left(\bar{\rho}_{i}\right)\right\rangle\right)$. The total average potential energy, $E$, is given by the sum of $E_{\text {peir }}$ and $E_{\text {emb }}=\Sigma F_{i}\left(\left\langle\left(\bar{\rho}_{i}\right)\right\rangle\right)$. We note that an expansion similar to equation (7) was recently used in a variational treatment of the free energy of pure metallic solids and liquids [12], with a similar conclusion as to the relative unimportance of the second-order term.

The calculation of the segregation around an interface is performed in steps. First, the properties of the perfect, uniform composition crystal are determined. This is done by choosing a composition and then minimizing the free energy, at the temperature and pressure of interest, with respect to the lattice parameter. Differentiating this equilibrium free energy with respect to composition gives the chemical potential difference $\Delta \mu$. For the $\mathrm{Cu}-\mathrm{Ni}$ alloy system discussed below, we have verified that the equilibrium structures are solid solutions at the temperature, pressure and composition of interest by minimizing the grand potential [equation (3)] with respect to lattice parameter and local concentration and verifying that the resultant concentration profile was uniform. This is in agreement with Monte Carlo simulations $[6,10]$ employing the same potentials. Since at equilibrium the chemical potential of a component is everywhere constant, we fix the chemical potentials at their bulk values, introduce the appropriate interface, and minimize the grand potential with respect to the concentration and position of each site.

The geometry of the cell used in the grainboundary simulations is the same as that employed in earlier studies on grain boundaries in pure systems $[2,3]$. As shown in Fig. 1, the simulation cell is divided into two regions, I and II. The effective atoms 
in region I are completely free to move in response to the forces due to other atoms and the concentration at each site is allowed to vary. The atoms in Region II, however, are constrained such that Region II is a perfect crystal with the lattice constant and average concentration on each site appropriate to the simulation temperature, pressure, and bulk concentration. The equilibrium configuration and concentrations of the effective atoms are obtained by minimizing equation (3) with respect to the coordinates, the site concentrations, and the relative positions of the upper and lower crystals $(4 N+3$ variables, where $N$ is the number of atoms in the system). Addition of the three variables corresponding to the relative positions of the two crystals removes all constraints on the relative in-plane translation of the two crystals and on the expansion in the $z$-direction, which may occur if the excess volume of the grain boundary is not zero. This type of simulation guarantees that a perfect crystal is at zero net stress. However, since periodic boundary conditions are imposed in the $x$ and $y$ directions and the size of the boundary in those directions is fixed by the lattice constant of the perfect system, some net stress may develop in the grain boundary region, which is appropriate since typical grain sizes are extremely large relative to the width of the grain boundary. The free-surface results were obtained by moving the two crystals far enough apart so that they did not interact with one another. The simulations then proceeded in the same way as for the grain boundaries.

\section{RESULTS}

The free energy minimization method, described above, was employed to study surface segregation and grain boundary segregation in the $\mathrm{Ni}-\mathrm{Cu}$ system. These cases were all examined in the solid solution region of the phase diagrams. These have all previously been studied by Monte Carlo (MC) methods $[6,7]$ using the same potentials as employed here. This was done in order to gauge the accuracy and utility of the present alloy simulation approach.

The point approximation for the entropy equation (2) implies that there is no correlation between the concentration at neighboring atomic sites. The internal energy term, equation (4), will lead to short range ordering. We can obtain an estimate of the accuracy of the configurational entropy point approximation assumption, at least for the bulk, by examining recent $\mathrm{MC}$ results [6] on the $\mathrm{Ni}-\mathrm{Cu}$ system. A measure of short range compositional order is given by the Warren-Crowley parameter $\alpha$, which is defined as

$$
\alpha_{i}=1-\left(\frac{p_{i}^{\mathrm{ab}}}{X_{\mathrm{b}}}\right)
$$

and measures the probability of finding neighboring atoms of the opposite (or the same) type around a site. In equation (9), the subscript $i$ represents the $i$ th

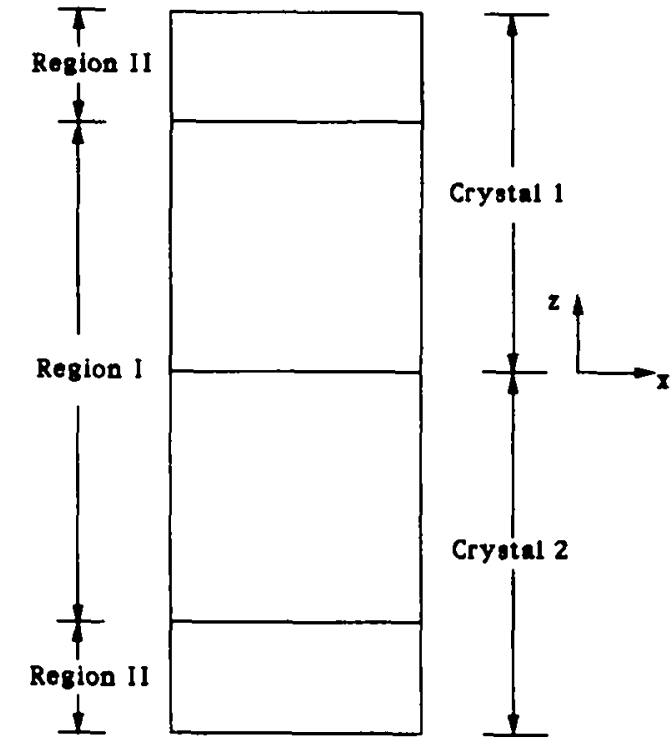

Fig. 1. The simulation cell employed in the present grain boundary simulations. The grain boundary is in the middle region $I$. The atoms in region I are free to move, while those in region II move rigidly with the rest of the crystal. Crystals in I and II are free to translate relative to one another. Periodic boundary conditions are employed in directions parallel to the interface.

shell of neighbors, $p_{i}^{\mathrm{ab}}$ is the probability that a specific atom in the $i$ th neighbor shell around an atom of type a will be of type $b$, and $X_{b}$ is the bulk concentration of type $b$. For a perfect solid solution with no short-range order, $p_{i}^{\mathrm{ab}}=X_{\mathrm{b}}$ and $\alpha_{i}=0$, which is what we find with our model for all bulk concentrations at this temperature. In the MC simulations, Foiles found [6] for the first neighbor shell around $\mathrm{Ni}$ atoms $\alpha$ values ranging from 0.149 at $X_{\mathrm{Cu}}=0.284$ to 0.085 at $X_{\mathrm{Cu}}=0.722$, which corresponds to a probability of finding a $\mathrm{Cu}$ atom around a $\mathrm{Ni}$ atom of 0.24 at $X_{\mathrm{Cu}}=0.284$ and 0.66 at $X_{\mathrm{Cu}}=0.722$. Thus, the Monte Carlo results show a slight tendency for atoms of the same type to cluster that is not seen in the model calculations. From the MC calculations, however, the values for $\alpha_{i}$ decreases rapidly with distance, so the ordering in these alloys is quite small and therefore we do not expect the point approximation for the configuration entropy to be too severe.

\subsection{Surface segregation in $\mathrm{Ni} / \mathrm{Cu}$ alloys}

We determined the structure and composition of three free surfaces in $\mathrm{Ni}-\mathrm{Cu}$ at a series of bulk concentrations at $800 \mathrm{~K}$. The same surfaces and EAM potentials [6] were employed in recent MC calculations. In the present study, we did not explicitly consider any surface reconstructions by restricting our initial structure to the unrelaxed $1 \times 1$ surfaces. The $\mathrm{Cu}$ composition of each layer $\left(X_{\text {kyer }}\right)$ was determined by averaging the concentration of $\mathrm{Cu}$ over all of the atomic sites in that layer (all concentrations will be given in atomic percent). The results are shown in Table 1, along with those reported by 
Table 1. Calculated composition profile in at.\% $\mathrm{Cu}$ for $\mathrm{Ni}-\mathrm{Cu}$ alloy surfaces at $800 \mathrm{~K}$

\begin{tabular}{lrrrrrr}
\hline Face & \multicolumn{1}{c}{$X_{\mathrm{Cu}}$} & \multicolumn{1}{c}{$X_{1}$} & \multicolumn{1}{c}{$\boldsymbol{X}_{2}$} & \multicolumn{1}{c}{$X_{3}$} & \multicolumn{1}{c}{$X_{4}$} & $\Sigma\left(X_{1}-X_{\mathrm{Cu}}\right)$ \\
\hline (111) & & & & & & \\
Present & 4.8 & 94.8 & 4.0 & 3.9 & 4.8 & 0.88 \\
MC & & 89.4 & 3.0 & 4.0 & 5.0 & 0.82 \\
Present & 29.2 & 96.7 & 11.9 & 15.0 & 23.1 & 0.30 \\
MC & & 95.0 & 11.0 & 19.1 & 27.1 & 0.35 \\
Present & 55.2 & 96.2 & 17.8 & 32.4 & 55.5 & -0.19 \\
MC & & 96.2 & 21.3 & 43.2 & 56.9 & -0.03 \\
Present & 71.9 & 97.4 & 46.1 & 71.5 & 71.9 & -0.01 \\
MC & & 97.5 & 39.0 & 70.2 & 76.8 & -0.04 \\
Present & 93.3 & 99.1 & 83.0 & 94.0 & 93.3 & -0.04 \\
MC & & 99.1 & 79.3 & 93.8 & 93.6 & -0.07 \\
(110) & & & & & & \\
Present & 29.1 & 99.7 & 37.8 & 13.1 & 15.1 & 0.49 \\
MC & & 99.2 & 36.8 & 14.1 & 19.8 & 0.53 \\
Present & 55.1 & 99.6 & 44.2 & 23.6 & 34.7 & -0.18 \\
MC & & 99.4 & 43.9 & 25.4 & 40.3 & -0.11 \\
Present & 72.0 & 99.7 & 58.0 & 46.7 & 64.8 & -0.19 \\
MC & & 99.7 & 55.8 & 44.3 & 64.8 & -0.23 \\
(100) & & & & & & \\
Present & 25.0 & 98.6 & 14.1 & 9.4 & 14.4 & 0.37 \\
MC & & 97.5 & 11.7 & 11.6 & 18.6 & 0.39 \\
Present & 57.0 & 98.3 & 21.3 & 25.0 & 48.5 & -0.35 \\
MC & & 98.1 & 22.7 & 36.6 & 57.7 & -0.13 \\
Present & 73.9 & 98.9 & 44.0 & 64.2 & 77.2 & -0.11 \\
MC & & 98.7 & 38.6 & 60.9 & 78.1 & -0.19 \\
\hline
\end{tabular}

The subscripts refer to the atomic layers numbered from the surface The last column is the sum of the deviations of the layer concentration from the bulk and is reported in terms of total number of excess monolayers. The summation was performed over the four atomic layers nearest the surface. The results marked MC are from Ref. [6]

Foiles from the MC calculations at the same concentrations. The agreement between the two sets of calculations is very good, with an average error in the concentration of the surface layer of less than $1 \%$. The surface layers of the (111), (110) and (100) surfaces are almost pure $\mathrm{Cu}$, nearly independent of $\mathrm{Cu}$ concentration in the bulk. In Fig. 2, we show the $\mathrm{Cu}$ concentrations of the individual atomic layers parallel to the surface, $X_{\text {leyer }}(\mathrm{Cu})$, as a function of the layer number for the (111) surface with a bulk concentration of $\mathrm{Cu}$ of $55.2 \%$. The agreement between the predicted segregation and the reported MC results is quite good, though we slightly overestimate the $\mathrm{Cu}$ depletion in layers 2 and 3 . Note that

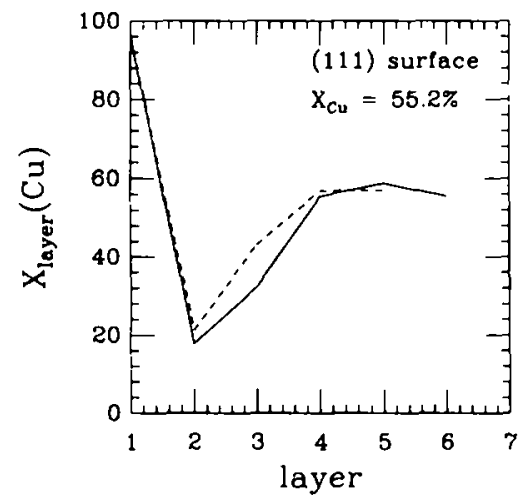

Fig. 2. Concentration of $\mathrm{Cu}$ atoms averaged over the layers parallel to the surface for a (111) surface in $\mathrm{Cu}-\mathrm{Ni}$ with 55.2 at. \% $\mathrm{Cu}, X_{\text {myer }}$, plotted as a function of layers away from the surface. The solid lines are the present results and the dashed lines are the results from MC calculations.

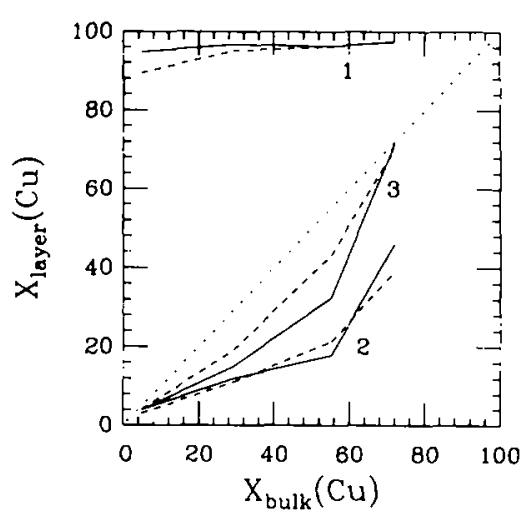

Fig. 3. Concentration of $\mathrm{Cu}$ atoms averaged over the layers parallel to the surface for a (I11) surface in $\mathrm{Cu}-\mathrm{Ni}, X_{\text {luyer }}$, plotted as a function of bulk $\mathrm{Cu}$ concentration, $X_{\text {bulk }}$. The solid lines are the present results and the dashed lines are the results from MC calculations. The short-dashed line would be the result for a bulk solution. Plotted are the results for the first three layers.

while the surface layer is very strongly enriched in $\mathrm{Cu}$ (relative to the bulk), the $\mathrm{Cu}$ concentration on the second layer is substantially depressed relative to the bulk $\mathrm{Cu}$ concentrations. Similar oscillatory segregation profiles are observed for all of the surfaces examined. Despite the large changes from the bulk values, the segregation is of a rather limited spatial extent; the bulk concentration value has been reached by essentially the 4 th atomic layer.

In Fig. 3, we show the $\mathrm{Cu}$ layer concentrations as a function of the bulk $\mathrm{Cu}$ concentration for the three (111) atomic layers closest to the surface. Deviations of these curves from the $X_{\text {layer }}=X_{\text {bulk }}$ line indicate segregation; if $X_{\text {layer }}$ is above that line, the $\mathrm{Cu}$ concentration is enhanced, if below, then the concentration of $\mathrm{Ni}$ is higher than in the bulk. Note that the $\mathrm{Cu}$ concentration in the first layer is considerably enhanced for all bulk concentrations, while for layers 2 and 3 , the $\mathrm{Ni}$ concentration is greater than in the bulk. A similar plot of the fourth and higher layers essentially follow the bulk concentration, the layer concentration has largely reached the bulk concentration by the fourth layer. Once again, agreement between the present simulations and the Monte Carlo data is excellent. We do, however, slightly overestimate the degree of segregation on the first and third layers. Even there, however, the overall trends in the layer concentrations are the same. Examination of the results for the other surfaces also indicates excellent overall agreement with the Monte Carlo data.

A measure of the net segregation to the surface can be obtained by summing the deviation of the layer concentrations $\left(X_{\text {layer }}\right)$ from the bulk value ( $\left.X_{\text {bulk }}\right)$, $\Sigma_{\text {layer }}\left(X_{\text {layer }}-X_{\text {bulk }}\right)$. These data are reported in Table 1 for all three surfaces and plotted in Fig. 4 for the (111) surface as a function of the bulk $\mathrm{Cu}$ concentration. As for the individual layer concentrations, the net segregation results are in reasonable agreement with the MC values. We see that at low 


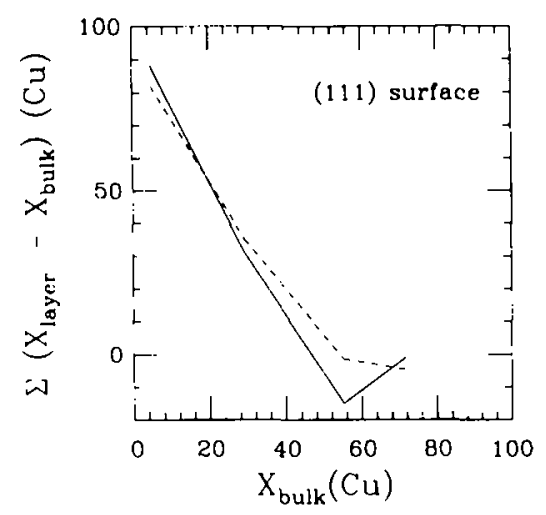

Fig. 4. Total net segregation of $\mathrm{Cu}$ to the surface $\left[\Sigma_{\text {layer }}\left(X_{\text {layer }}-X_{\text {bulk }}\right)\right]$ as described in the text plotted as a function of bulk $\mathrm{Cu}$ concentration, $X_{\text {bulk }}$. The solid lines are the present results and the dashed lines are the results from MC calculations.

bulk $\mathrm{Cu}$ concentrations, there is considerable $\mathrm{Cu}$ segregation to the surface region. As the bulk $\mathrm{Cu}$ concentration increases, the net segregation decreases. This is simply a result of the fact that the surface $\mathrm{Cu}$ concentration is nearly $100 \%$, independent of bulk $\mathrm{Cu}$ concentration. Figure 4 and Table 1 show that when the bulk $\mathrm{Cu}$ concentration becomes large, there is a net Ni segregation to the region. The $\mathrm{Ni}$ segregation to the surface in $\mathrm{Cu}$ rich alloys is, however, much weaker than the $\mathrm{Cu}$ segregation to the surface in the $\mathrm{Ni}$ rich alloys. This result appears to be independent of surface plane.

\subsection{Grain boundary segregation in $\mathrm{Ni}-\mathrm{Cu}$ alloys}

Calculations were performed on three [100] twist grain boundaries in $\mathrm{Ni}-\mathrm{Cu}$ alloys at $800 \mathrm{~K}$ for a series of bulk $\mathrm{Cu}$ concentrations. These conditions are identical to those employed in the free-surface studies discussed above. The grain boundaries were created by cutting a single crystal along an (100) plane and rotating the resultant two crystals relative to one another around a common [100] axis by $36.9^{\circ}, 22.6^{\circ}$, and $10.4^{\circ}$, which correspond to the $\Sigma 5, \Sigma 13$, and $\Sigma 61$ [100] twist boundaries, respectively (the $\Sigma$ value is equal to the reciprocal of the coincident site lattice density). The $\mathrm{Ni}-\mathrm{Cu}$ bulk alloy compositions em. ployed in these calculations were 10,50 and $90 \% \mathrm{Cu}$. These particular choices for the grain boundaries, bulk compositions and temperature were made in order to compare with the Monte Carlo simulation results reported by Foiles [7] using the same EAM potentials as those employed here [6]. Since we do not require as large of a sample in order to get statistically significant data as in the Monte Carlo simulations, we are able to use relatively small simulation cells (in the plane of the boundary). In the present calculations, we used $2 \times 2$ arrays of the basic periods of the $\Sigma 5$ and $\Sigma 13$ boundaries and a single unit cell for the $\Sigma 61$ boundary.

In Table 2 we compare the concentration profiles obtained from our calculations with the MC data.
Table 2. Calculated composition profile in at.\% $\mathrm{Cu}$ for $\mathrm{Ni}-\mathrm{Cu}$ grain boundaries at $800 \mathrm{~K}$

\begin{tabular}{lllllll}
\hline Boundary & $X_{0}$ & $X_{1}$ & $X_{2}$ & $X_{3}$ & $X_{4}$ & $\Sigma\left(X_{1}-X_{C_{u}}\right)$ \\
\hline$\Sigma 5$ & & & & & & \\
Present & 90 & 96.2 & 87.2 & 89.0 & 90.3 & 0.05 \\
MC & & 95 & 90 & 90 & & 0.10 \\
Present & 50 & 82.2 & 41.8 & 37.0 & 45.9 & 0.22 \\
MC & & 78 & 48 & 42 & & 0.36 \\
Present & 10 & 85.6 & 25.8 & 10.1 & 9.3 & 1.83 \\
MC & & 74 & 22 & 11 & & 1.54 \\
$\Sigma 13$ & & & & & & \\
Present & 90 & 95.3 & 89.1 & 89.2 & 90.0 & 0.07 \\
MC & & 95 & 90 & 90 & & 0.10 \\
Present & 50 & 78.8 & 48.1 & 50.0 & 50.0 & 0.54 \\
MC & & 74 & 52 & 44 & & 0.40 \\
Present & 10 & 79.4 & 26.2 & 10.0 & 9.2 & 1.71 \\
MC & & 62 & 24 & 11 & & 1.34 \\
$\Sigma 61$ & & & & & & \\
Present & 90 & 92.9 & 90.7 & 89.9 & 90.0 & 0.07 \\
MC & & 92 & 91 & 90 & & 0.06 \\
Present & 50 & 71.5 & 56.2 & 48.0 & 47.1 & 0.51 \\
MC & & 66 & 57 & 51 & & 0.48 \\
Present & 10 & 65.1 & 32.3 & 14.2 & 10.1 & 1.63 \\
MC & & 41 & 27 & 15 & & 1.06 \\
\hline
\end{tabular}

The subecripts refer to the atomic layers numbered from the boundary. The last column is the sum of the deviations of the layer concentration from the bulk and is reported in terms of total number of excess monolayers. In all caces, the summation was performed over three layers on each side of the boundary. The results marked MC are from Ref. [7].

The concentration of $\mathrm{Cu}$ on each atomic layer $X_{\text {lyyer }}(\mathrm{Cu})$ is plotted as a function of layer number (where the grain boundary lies between layers 0 and 1) in Figs $5-7$ for the $\Sigma 5, \Sigma 13$, and $\Sigma 61$ [100] twist boundaries, respectively. The layer concentrations were determined by averaging over all of the atomic sites on the appropriate (002) planes. Comparison of our results (solid lines) with MC results (dashed lines) shows good overall agreement, especially for the larger bulk $\mathrm{Cu}$ concentrations. There is a tendency for the model to overestimate the amount of $\mathrm{Cu}$ that segregates to the atomic layer adjacent to the boundary; nonetheless, the only significant disagreement between our results and the MC data is at low bulk $\mathrm{Cu}$ concentration $(10 \%)$. For these low bulk $\mathrm{Cu}$ concentrations, the results become worse as the grainboundary angle becomes smaller (i.e. our results for

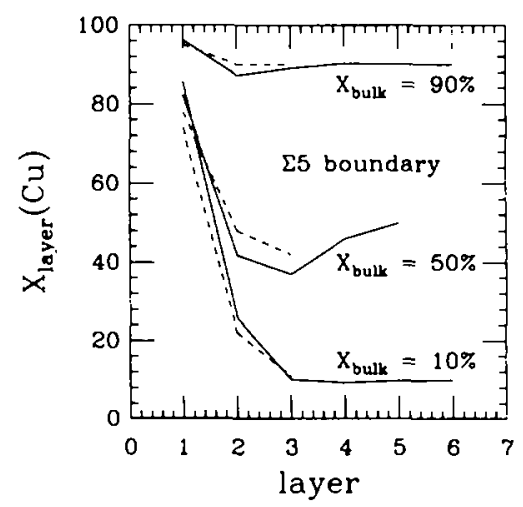

Fig. 5. Concentration of $\mathrm{Cu}$ atoms averaged over the layers parallel to a $\Sigma 5[100]$ twist boundary, $X_{\text {myen }}$, in $\mathrm{Cu}-\mathrm{Ni}$ with 10,50 , and 90 at. $\%$ bulk $\mathrm{Cu}$ plotted as a function of layers away from the surface. The solid lines are the present revults and the dashed lines are the results from MC calculations. 


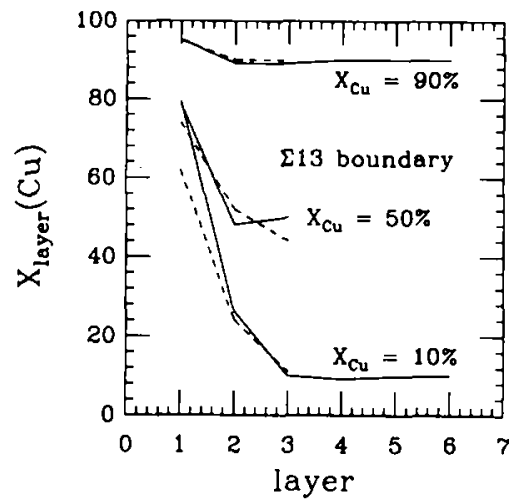

Fig. 6. Concentration of $\mathrm{Cu}$ atoms averaged over the layers parallel to a $\Sigma 13[100]$ twist boundary, $X_{\text {nyer }}$, in $\mathrm{Cu}-\mathrm{Ni}$ with 10,50 , and 90 at. $\%$ bulk $\mathrm{Cu}$ plotted as a function of layers away from the surface. The solid lines are the present results and the dashed lines are the results from MC calculations.

$\Sigma 5$ are in better agreement with the MC data than the $\Sigma 13$ results, which are better than for $\Sigma 61$ ). However, even in the worst cases (lowest $\mathrm{Cu}$ concentration and lowest boundary misorientation angle) the model still produces the proper trends and is still at least semiquantitative.

As in the MC studies, we see a very strong segregation of $\mathrm{Cu}$ at the atomic layer adjacent to the grain boundary, especially at low bulk $\mathrm{Cu}$ concentrations. While the second and third atomic layers from the free surfaces tend to be depleted of $\mathrm{Cu}$, this effect is not generally present for these grain boundaries. The shortest period grain boundary (the $\Sigma 536.9^{\circ}$ boundary) does, however exhibit this $\mathrm{Cu}$ depletion/ $\mathrm{Ni}$ enrichment on the second and third layers in all but the most Ni-rich alloy, as shown in Fig. 8.

The net grain boundary segregation may be obtained by summing the deviation of the layer concentrations from the bulk value, $\left(\Sigma_{\text {layer }}\left[X_{\text {Lyer }}-X_{\text {bulk }}\right]\right)$. Unfortunately, the MC data was only presented for the first three layers. In many cases (see Table 2), the layer concentrations have not converged to the bulk

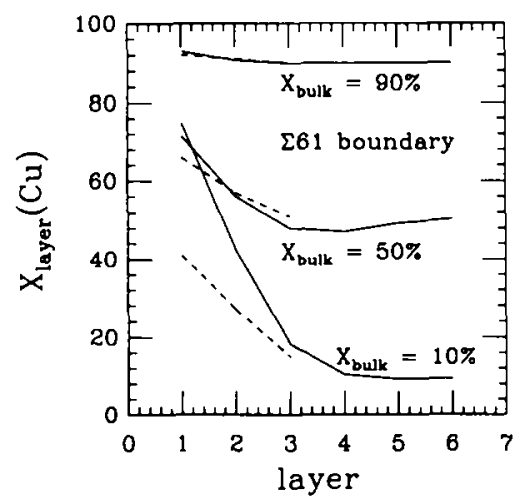

Fig. 7. Concentration of $\mathrm{Cu}$ atoms averaged over the layers parallel to a $\Sigma 61$ [100] twist boundary, $X_{\text {tayer }}$, in $\mathrm{Cu}-\mathrm{Ni}$ with 10,50 , and 90 at.\% bulk $\mathrm{Cu}$ plotted as a function of layers away from the surface. The solid lines are the present results and the dashed lines are the results from MC calculations.

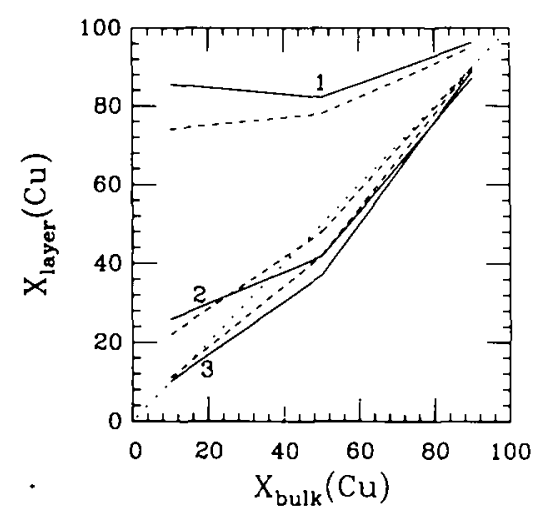

Fig. 8. Concentration of $\mathrm{Cu}$ atoms averaged over the layers parallel to a $\Sigma 5[100]$ twist boundary, $X_{\text {lyee }}$, plotted as a function of bulk $\mathrm{Cu}$ concentration, $X_{\text {bulk }}$. The solid lines are the present results and the dashed lines are the results from MC calculations. Plotted are the first three layers.

values by this distance from the grain boundary. Thus, the MC results do not provide an accurate estimate of the net boundary segregation. Nonetheless, in order to make the most appropriate comparison between the present results and the MC data, we have calculated the net grain boundary segregation in all cases to three atomic layers on either side of the grain boundary. The agreement between the present simulation results and the MC data for the net grain boundary segregation is significantly worse than for the free surfaces. On average the error is approximately $30 \%$, with a worst case error of $50 \%$ and a best case of $7 \%$. In general the error increases with increasing $\mathrm{Cu}$ concentration in the bulk and decreasing boundary period (i.e. decreasing $\Sigma$ value). In Fig. 9, we plot the net segregation results for the $\Sigma 5$ boundary as a function of bulk composition. Unlike the free-surface, this function is always positive, indicating that there is always a net segregation of $\mathrm{Cu}$ to the grain boundaries. As with all of the other major trends, the present segregation results are consistent with the Monte Carlo results.

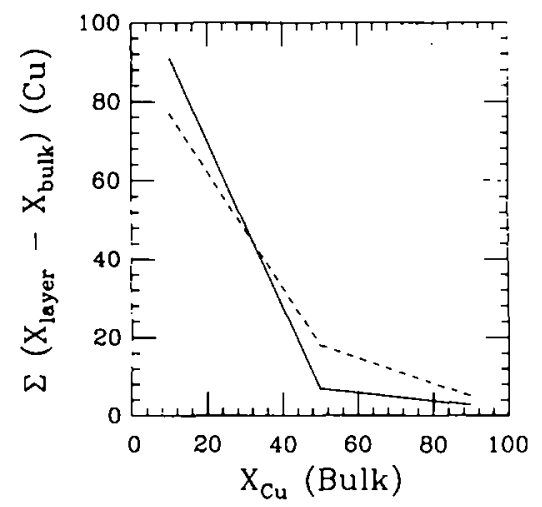

Fig. 9. Total net segregation of $\mathrm{Cu}$ to a $\Sigma 5$ boundary $\left[\Sigma_{\text {kyout }}\left(X_{\text {leyer }}-X_{\text {bulk }}\right)\right]$ as described in the text plotted as a function of bulk $\mathrm{Cu}$ concentration, $X_{\text {bulk. }}$. The solid lines are the present results and the dashed lines are the results from MC calculations. 
In Fig. 10, we show the average concentration of $\mathrm{Cu}$ atoms per site for the first three layers on either side of the $\Sigma 61$ boundary with $10 \% \mathrm{Cu}$ in the bulk, which may be compared with the $\mathrm{Cu}$ concentration contour plots for this grain boundary under the same conditions in Fig. 2 of Ref. [7] by Foiles. Layer 1 [Fig. 10(a)] shows that $\mathrm{Cu}$ segregations is strongest along the square pattern that corresponds to the location of screw dislocations in this relatively low angle boundary. The $\mathrm{Cu}$ concentration is lowest at the center of the square pattern, which is furthest from the dislocation lines. In contrast, layers 2 and 3 show a decided segregation of $\mathrm{Cu}$ to the center and corners of the cell with some $\mathrm{Cu}$ depletion in the vicinity of the positions of the screw dislocations. These patterns are in nearly identical to those obtained by Foiles using Monte Carlo simulations. The relative magnitudes of the maxima and minima in the concentrations obtained in the present study and those obtained by $\mathrm{MC}$ are essentially the same. The absolute magnitudes of the $\mathrm{Cu}$ concentrations, however, are too large by a factor of 1.7 in layer $\mathbf{1}$, 1.8 in layer 2 and 1.6 in layer 3.

Since the present simulations employing the free energy minimization method are very efficient, it is possible to perform more complete studies than easily possible with MC methods. In Fig. 11, we show the results of such a study, where we examine the temperature-dependence of the layer concentrations at a $\Sigma 5$ [001] twist grain boundary for $50 \%$ bulk $\mathrm{Cu}$. (These calculations were performed with a more current version of the EAM potentials [11] than used in the MC study, so the results are somewhat different than those reported in Table 2.) We see that the dominant effect of temperature is to decrease the amount of segregation to the boundary region. This decrease in segregation is due primarily to the configurational entropy, which drives the system to a completely uniform state. The segregation to layer 1 is the most affected by temperature since the segregation to that layer is the most pronounced. On energetic grounds, we know that at $0 \mathrm{~K}$ there would be complete segregation to the boundary, as discussed in detail below. At high temperatures, the configurational entropy term becomes important, thereby leading to a more uniform/disordered state. For the layers farther from the boundary, the results are essentially the same.

The fundamental quantity from which all thermodynamic information can be derived is the free energy. For a defect, one can define an excess free energy for the defect as the difference in free energies between the system with the defect and the perfect system. Here, we use the grand potential [equation

Fig. 10. Distribution of $\mathrm{Cu}$ at a $\Sigma 61$ [100] twist boundary for $10 \%$ bulk $\mathrm{Cu}$ in a $\mathrm{Ni}-\mathrm{Cu}$ alloy at $800 \mathrm{~K}$. The circles are atomic sites and the darker the circle, the higher the $\mathrm{Cu}$ concentration at that site. Shown are (a) layer 1, (b) layer 2 , and (c) layer 3 .
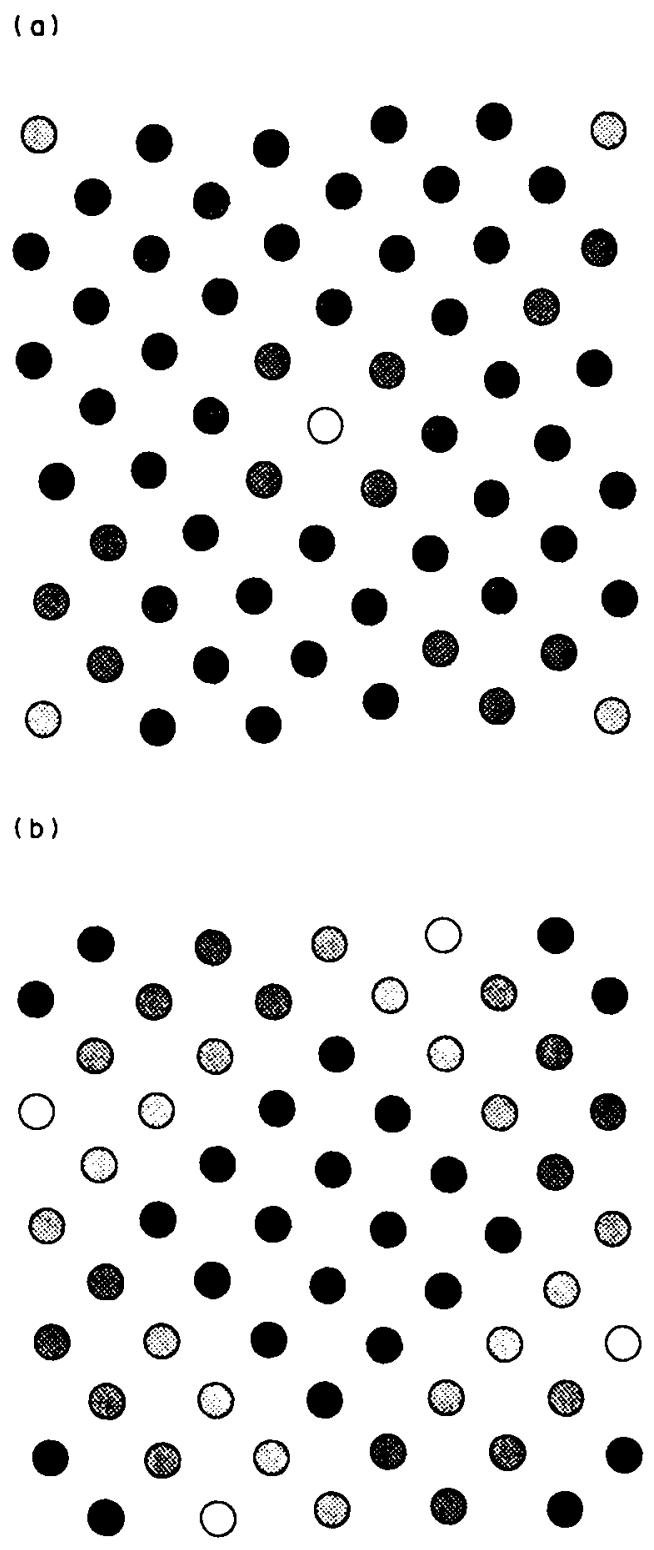

(c)

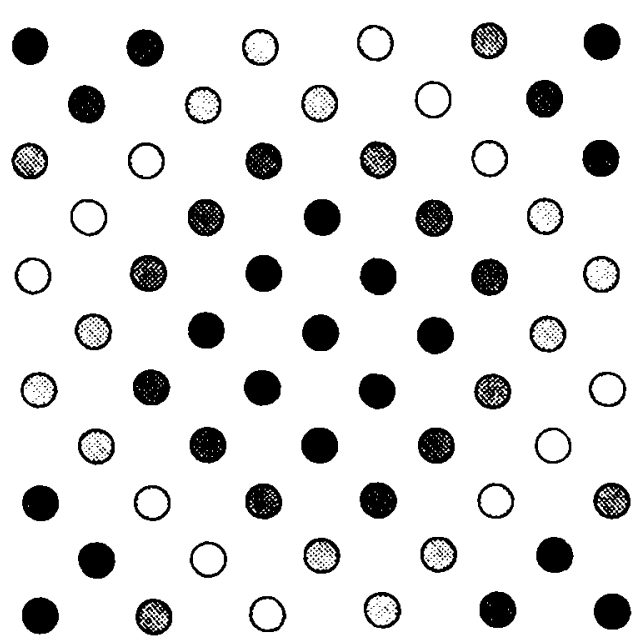




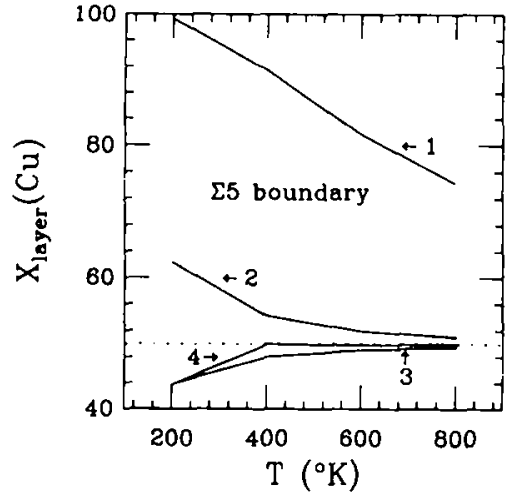

Fig. 11. Temperature dependence of the concentration of $\mathrm{Cu}$ atoms averaged over the layers parallel to a $\Sigma 5[100]$ twist boundary, $X_{\text {byer }}$, for a system with $50 \%$ bulk Cu. Shown are the resuits for the first 4 layers.

(9)] and define $\Gamma_{\mathrm{zb}}$ as the excess free energy for the grain boundary. The excess grain boundary free energy has contributions from the grain boundary itself, the non-uniform composition profile near the grain boundary and the interaction between them. We plot that excess free energy (calculated with the EAM potentials of Ref. [11]) in Fig. 12 as a function of bulk $\mathrm{Cu}$ concentration at $800 \mathrm{~K}$ for the three boundaries studied here. The free energy of [001] symmetric twist boundaries in elemental metals tends to increase in a nearly monotonic fashion with increasing misorientation angle from a low at $0^{\circ}$ to a high at $45^{\circ}$ and the free energy versus misorientation plot is symmetric about $45^{\circ}$. The ordering of the three curves in Fig. 12 is as expected with the largest misorientation angle boundary $\Sigma 5$ highest and the lowest angle boundary $\Sigma 61$ lowest. The effect of composition on the excess grain boundary free energy is to smoothly interpolate between the grain boundary values in the pure solids. The effect of temperature on $\Gamma_{z b}$ is shown in Fig. 13 for the $\Sigma 5$ boundary at three different bulk compositions. There we see quite different behavior for the three boundaries. The excess grain boundary free energy values for $90 \%$ bulk $\mathrm{Cu}$ are quite typical of the temperature-

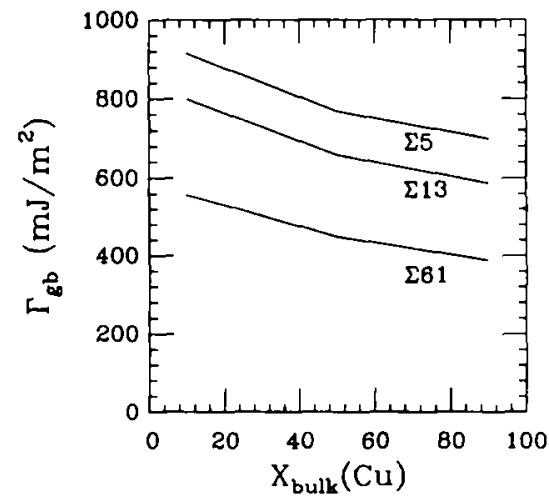

Fig. 12. Excess grain boundary free energy, $\Gamma_{\text {pto }}$, as defined in the text, as a function of bulk $\mathrm{Cu}$ concentration, $X_{\text {bulk }}$, for the $\Sigma 5, \Sigma 13$, and $\Sigma 61$ [100] twist boundaries.

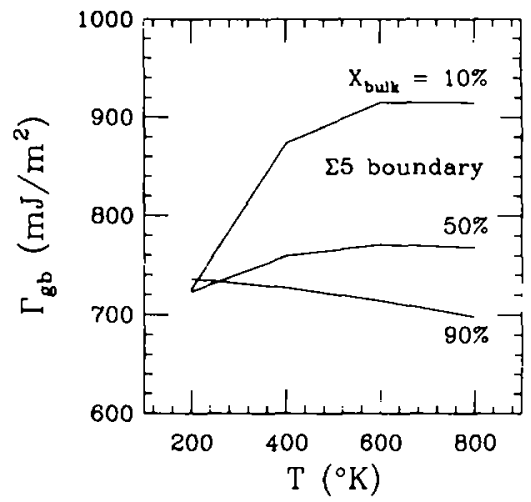

Fig. 13. Excess grain boundary free energy, $\Gamma_{\text {sb }}$, as defined in the text, as a function of temperature for the $\Sigma 5$ [100] twist boundary. Shown are the results for 10, 50, and 90 at. $\% \mathrm{Cu}$ in the bulk.

dependence of the grain boundary free energy in a pure system; the grain-boundary free energies decreases with temperature due to entropy effects. For 50 and $10 \%$ bulk $\mathrm{Cu}$, however, the situation is quite different; $\Gamma_{\mathrm{gb}}$ shows a very strong increase with $T$ that levels off around $600 \mathrm{~K}$. Since $\mathrm{Cu}$ strongly segregates to the grain boundary at low temperature, the presence of $\mathrm{Cu}$ at the grain boundary clearly lowers the energy of the system. When the temperature is increased, the configurational entropy drives the $\mathrm{Cu}$ off the boundary, thereby raising the energy (which more than balances the entropy effect on the grand potential). This effect is much more pronounced with decreasing bulk $\mathrm{Cu}$ concentration because it is at low bulk $\mathrm{Cu}$ concentration that segregation is the strongest. For instance, for the boundary in the $50 \%$ bulk Cu system, the net segregation is 75 at $200 \mathrm{~K}$ and 26 at $800 \mathrm{~K}$. The leveling off of $\Gamma_{\mathrm{gb}}$ at high temperatures may be attributed to the increase in the vibrational entropy term.

\section{DISCUSSION}

The overall agreement between the present results on segregation to free surfaces and grain boundaries in metals and accurate Monte Carlo data is striking, considering the simplicity of the model and the ease and efficiency of the present method. In most cases, the model gives quantitative agreement with the MC results $[6,7]$ and, in all cases, the qualitative trends predicted by the model are correct. Even where the greatest discrepancies between the Monte Carlo and the present results exist, the two methods yield segregation which seldom differ by more than a factor of two. Successes of the model include the accurate prediction of almost complete $\mathrm{Cu}$ segregation to, and $\mathrm{Ni}$ enhancement just below, surfaces in $\mathrm{Cu}-\mathrm{Ni}$ alloys as well as the prediction of a net $\mathrm{Ni}$ enhancement for bulk $\mathrm{Cu}$ concentrations greater than about $50 \%$. The method also does well in predicting the segregation in grain boundaries in $\mathrm{Cu}-\mathrm{Ni}$ alloys. The agreement with MC data [7] is, for the most part, excellent for 
bulk $\mathrm{Cu}$ concentrations of 50 and $90 \mathrm{at} \%$, though the results at $10 \%$ are less good. In all cases, however, we overestimate the $\mathrm{Cu}$ segregation right at the boundary. Thus, the model, for all its simplicity and approximated nature, is accurate enough to serve as a predictive tool.

Nonetheless, the discrepancies between the present results and the Monte Carlo data should be addressed. The key to understanding these deviations lies in the central approximation in our model; that the concentrations at any site are completely uncorrelated with those at any other site. This approximation enters into the model in two central ways. First, the expression we employ for the configurational entropy [equation (2)] is valid only in the limit of uncorrelated concentrations. Second, the mean-field expressions used for the average potential energy are also derived based on the assumption of uncorrelated sites.

The configurational entropy in equation (2) has a very simple form and reaches its maximum value at a concentration of $50 \%$. Since the expression is derived based on the assumption of uncorrelated sites, any correlation in the real system will produce an entropy that is less than obtained from equation (2), i.e. our expression for the entropy is an upper bound on the true configurational entropy. An overestimate of the configurational entropy will, therefore, tend to produce an underestimate of the true interfacial segregation. This, however, is not observed in the present calculations in the $\mathrm{Cu}-\mathrm{Ni}$ system. Therefore, we conclude that the mean-field expression for the potential energy [equations (6-8)] is the dominant source of error in this method. Nonetheless, the simple point approximation for the entropy is known to produce problems when studying the details of configurational entropy driven phase transformations.

The errors in the mean-field expression for the energy are difficult to determine precisely. The general effect is, however, easily understood. Suppose that we have a system that contains, on average, $50 \%$ of each element, say $a$ and $b$, that interact with pair potentials, $\phi$. The mean-field expression for the energy would be

$$
U=0.5^{2} \phi_{\mathrm{aa}}+0.5^{2} \phi_{\mathrm{bb}}+2\left(0.5^{2}\right) \phi_{\mathrm{ab}} .
$$

Further, suppose that while on average every site has equal probability of being occupied by an a or b, there is a greater probability for two a atoms to occupy adjacent sites than an $a$ and $b$. In this case, it is clear that the mean-field prediction for the energy would be in error; the appropriate prefactor of $\phi_{\mathrm{aa}}$ would be greater than the $0.5^{2}$ in the above expression. Neglecting this correlation will create an error in the average potential energy which increases with increasing correlation. It is important to recognize that the type of correlation that causes this type of error is a temporal-spatial correlation. For example, if a given site is always occupied by an a atom and its neighbor site is always occupied by a b atom, this spatial correlation does not produce an error in the energy. Therefore, there is no inherent problem in using this approach to simulate an ordered alloy. At this stage, it remains unclear how to improve upon the simple mean-field picture of an atom while still retaining the simplicity necessary for efficient atomistic simulations.

The free energy simulation method, which we have described for binary alloys, may be extended to account for an arbitrary number of constituents. This may be accomplished by extending the "effective atom" concept to include all constituents. The composition of each atom will then be expressed in $n-1$ variables, where $n$ is the number of constituents. The configurational entropy term [equation (2)] can be generalized by summing the $c_{x}(i) \ln \left[c_{x}(i)\right]$ terms over all $1 \leqslant i \leqslant N$ atoms and over all $1 \leqslant \alpha \leqslant n$ constituents. In this case, the grand potential minimization requires the specification of $n-1$ chemical potentials and is performed over $3(n-1)(N-1)$ variables, accounting for the composition of each "atom". This approach may also be extended to include vacancies as one of the constituents. Minimization of the grand potential for the crystal with respect to "concentration" should yield the equilibrium vacancy concentration. However, since vacancy concentrations are so small this presents numerical difficulties. Work is currently underway to incorporate vacancies as a constituent.

The present simulation approach has two major advantages over competing simulation methods. They are: (1) the ability to provide thermodynamic data (e.g. free energy and entropy) and (2) computational efficiency. While the present simulations were performed on inexpensive computer workstations, the Monte Carlo simulation, with which these results were compared, required substantial quantities of supercomputer time. Efficient computation is not simply an economic issue; efficient methods are required to study trends in properties instead of isolated examples. Since the present simulation approach is based upon a form of direct free energy minimization, the free energy, and hence all other thermodynamic properties, are obtained as a simple by-product of determining the equilibrium atomic arrangements. Free energy may also be obtained from Monte Carlo simulations. Unfortunately, many Monte Carlo simulations must be performed in order to obtain the free energy for just one set of conditions. In fact, due to the extreme computational demand required to obtain alloy free energies from Monte Carlo data, we know of no cases in which the Monte Carlo method was employed to calculate the thermodynamic properties (free energies, etc.) of any alloy system.

In the present study, we have focussed on the application of our free energy simulation methods to grain boundaries and surfaces in alloys. Clearly, this method is much more general than the applications presented herein suggest. This method may be em- 
ployed with even greater efficiency to crystal properties, such as: lattice parameter vs composition and temperature, elastic constants vs composition and temperature, coefficient of thermal expansion vs composition and temperature, free energy (and those thermodynamic functions derivable from it) vs composition and temperature, etc. The application of these methods to other defects such as vacancies, stacking faults, dislocations, anti-phase boundaries, substitutional atoms, etc. represent straight-forward extensions of this method. In addition, properties such as brittle fracture toughness and diffusivity may be estimated based upon the defect properties determined with this method. We believe that the free energy simulation methods, described above, eliminate essentially all of the major difficulties inherent in simulating alloy systems. Given this conclusion, we believe then that the limiting step in obtaining accurate results from alloy simulations is the quality and availability of appropriate interatomic potentials.

\section{SUMMARY}

We have presented a new, accurate method for determining equilibrium segregation to defects in solids. This method is based upon a point approximation for the configurational entropy, an Einstein model for vibrational contributions to the free energy and may be employed with any type of description of atomic interactions. The equilibrium atomic structure, segregation and thermodynamics of a defect in an alloy is determined by minimizing the free energy with respect to atomic coordinates and composition of each site at constant chemical potential. In order to test the accuracy of this approach, we have compared our results with accurate Monte Carlo determinations and find good overall agreement for segregation to free surfaces and grain boundaries in
$\mathrm{Cu}-\mathrm{Ni}$ alloys. One of the main advantages this new method enjoys over other methods such as Monte Carlo, is the efficiency with which the atomic structure of a defect, segregation and thermodynamic properties can be determined. This efficiency is obtained in the framework of a very straightforward method and with little loss in accuracy.

Acknowledgements-We gratefully acknowlodge the Division of Materials Science of the Office of Basic Energy Sciences of the United States Department of Energy (DOE BES DMS), Grant No. FG02-88ER45367 for its support of this work. The work of R.L. was performed under the auspices of the U.S. Department of Energy and was also supported, in part, by DOE BES DMS.

\section{REFERENCES}

1. R. LeSar, R. Najafabadi and D. J. Srolovitz, Phys. Rev. Lett. 63, 624 (1989).

2. R. Najafabadi, D. J. Srolovitz and R. LeSar, J. Mater. Res. 5, 2663 (1990).

3. R. Najafabadi, D. J. Srolovitz and R. LeSar, J. Mater. Res. In press.

4. V. Kumer, Phys. Rev. B 23, 3756 (1981).

5. H. J. Leamy, G. H. Gilmer, K. A. Jackson and P. Bennema, Phys. Rev. Lett. 30, 601 (1973).

6. S. M. Foiles, Phys. Rev. B 40, 11502 (1989).

7. S. M. Foiles, Phys. Rev. B 32, 7685 (1985).

8. R. Kikuchi and J. Cahn, Phys. Rev. B 21, 1893 (1980); Mater. Res. Soc. Symp. 122, 231 (1988); Mater. Res. Soc. Symp. 122, 163 (1988).

9. H. A. Bethe, Proc. R. Soc. 150, 552 (1935).

10. M. S. Daw and M. I. Baskes, Phys. Rev. Lett. 50, 1285 (1983); Phys. Rev. B 29, 643 (1984).

11. S. M. Foiles, M. S. Daw and M. I. Baskes, Phys. Rev. $B$ 33, 7983 (1986).

12. R. LeSar, R. Najafabadi and D. J. Srolovitz, J. chem. Phys. 94, 5090 (1991).

13. W. Shockley, J. chem. Phys. 6, 130 (1938).

14. K. Huang, Statistical Mechanics. Wiley, New York (1963).

15. M. Lundberg, Phys. Rev. B 36, 4692 (1987).

16. J. H. Rose, J. R. Smith, F. Guinea and J. Ferrante, Phys. Rev. B 29, 2963 (1984). 